\title{
Research Article \\ Efficacy of Two-Stage Treatment of Onychomycosis Using a Long-Pulsed Nd:YAG 1064-nm Laser
}

\author{
Shan Zhong ${ }^{(D)},{ }^{1}$ Guan-ting Lin $\left.{ }^{(D}\right)^{2}$ and Jun-ying Zhao ${ }^{1}{ }^{1}$ \\ ${ }^{1}$ Department of Dermatology, Beijing Friendship Hospital, Capital Medical University, Beijing 100050, China \\ ${ }^{2}$ Department of Dermatology, Longyan First Hospital, Fujian Medical University, Longyan, Fujian 364000, China \\ Correspondence should be addressed to Jun-ying Zhao; zjy63014411@aliyun.com
}

Received 18 February 2019; Accepted 10 June 2019; Published 16 June 2019

Academic Editor: Laura De Martino

Copyright (c) 2019 Shan Zhong et al. This is an open access article distributed under the Creative Commons Attribution License, which permits unrestricted use, distribution, and reproduction in any medium, provided the original work is properly cited.

\begin{abstract}
Objectives. Onychomycosis is a fungal infection of the nail bed, nail matrix, and nail plate. Lasers have recently been studied as future clinical options for onychomycosis. We sought to evaluate the efficacy of the long-pulsed Nd:YAG 1064-nm laser on onychomycosis using a two-stage treatment. Methods. A total of 100 affected nails ( 88 toenails and 12 fingernails from 22 patients) were treated using a long-pulsed Nd:YAG 1064-nm laser. The self-controlled treatment schedule included the first stage (1 session per week for 8 weeks) and the second stage ( 1 session every 4 weeks for 16 weeks). Patients were followed up with for 12 weeks after the last laser treatment. Direct microscopy, cultures, and clinical assessments were performed at various time points. Results. Of the nails studied, $84 \%$ were infected by Trichophyton rubrum, while others were coinfected with T. rubrum and other fugal strains. The mycological clearance rate and the clinical efficacy rate of the nails were $29 \%$ and $21 \%$ after the first stage, $69 \%$ and $35 \%$ after the second stage, and $67 \%$ and $39 \%$ during follow-up, respectively. The second stage of laser treatment significantly improved the mycological clearance and clinical efficacy. Conclusion. Long-pulsed Nd:YAG 1064-nm laser two-stage treatment was effective for onychomycosis, with significantly improved mycological clearance and clinical efficacy. This trial is registered with ChiCTR 1900021669.
\end{abstract}

\section{Introduction}

Onychomycosis is a chronic fungal infection of the nail plate, nail matrix, and nail bed that is more common in the toenails. The prevalence of onychomycosis is $2-13 \%$ [1], and up to $14-28 \%$ among elderly patients over 60 years old [2]. Onychomycosis can cause pain, discomfort, and cosmetic concerns and thus significantly impacts patients, both physically and emotionally [3]. Although there have been advances in current treatment options, including terbinafine and itraconazole, that have improved the complete cure rate to $70.5 \%$ [2], recurrence (relapse or reinfection) still occurs in 10-53\% of patients [4]. Oral antifungal medications are associated with side effects, such as gastrointestinal upset, headaches, and liver dysfunction. Recently, laser treatments, including the use of the Nd:YAG 1064-nm laser, have shown encouraging and promising results in patients with onychomycosis [5-7]; however, there is no standard treatment course for laser treatment. Here, we report that a two-staged long-pulsed Nd:YAG 1064-nm laser treatment significantly improves onychomycosis.

\section{Methods}

2.1. Subjects. For this study, 22 patients ( 18 to 76 years old) with clinically and mycologically (both direct microscopy and cultures) diagnosed onychomycosis for at least one year were enrolled in the Department of Dermatology at Beijing Friendship Hospital. The Scoring Clinical Index of Onychomycosis (SCIO) [8] was 6-15 (grade II-IV). The study was approved by the Hospital Ethic Committee, and all patients signed written informed consent statements before the study started. Exclusion criteria included application of topical antifungal agents within the last months; application of systemic antifungal agents within the last 6 months; other nail diseases, such as lichen planus, psoriasis, atopic dermatitis, subungual hematoma, nevoid formation, or bacterial nail infections; pregnancy or lactation; and photohypersensitivity.

2.2. Treatment Procedure. Patients were treated with a longpulsed Nd:YAG 1064-nm laser (Dualis SP; Fotona, Slovenia). 


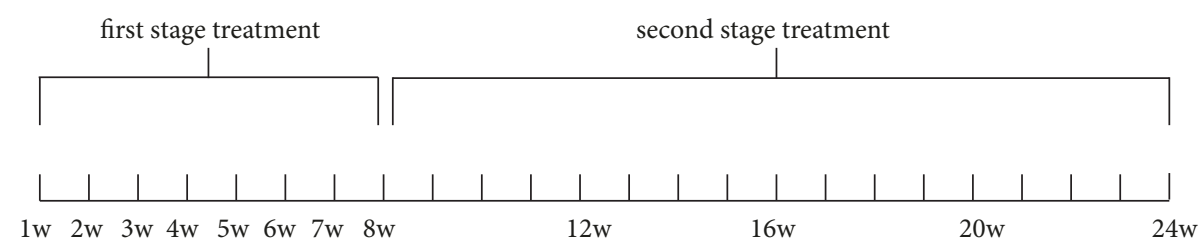

FIGURE 1: Two-stage treatment procedure. The entire procedure lasted 24 weeks.

TABLE 1: Nail characteristics.

\begin{tabular}{lcc}
\hline & & $\mathrm{N}(\%)$ \\
\hline Clinical type & DLSO & $87(87 \%)$ \\
& SWO & $(3 \%)$ \\
& PSO & $(2 \%)$ \\
& TPO & $2(2 \%)$ \\
SCIO grade & II & $17(17 \%)$ \\
& III & $55(55 \%)$ \\
& IV & $28(28 \%)$ \\
Hyperkeratosis & $<1 \mathrm{~mm}$ & $35(35 \%)$ \\
& $1-2 \mathrm{~mm}$ & $44(44 \%)$ \\
& $>2 \mathrm{~mm}$ & $21(21 \%)$ \\
\hline
\end{tabular}

DLSO: distal and lateral subungual onychomycosis, SWO: superficial white onychomycosis, PSO: proximal subungual onychomycosis, and TDO: total dystrophic onychomycosis.

The device fluency was set at $35-40 \mathrm{~J} / \mathrm{cm}^{2}$, with a pulse duration of $35 \mathrm{~ms}$, spot size of $4 \mathrm{~mm}$, and frequency of $1.0 \mathrm{~Hz}$. The fluency was chosen based on nail thickness, since thicker nails would require higher fluency. Each infected nail was treated with laser beam irradiation on the full nail plate and nail fold, using a spiral pattern that was repeated for 3 passes, with 2-min pause between passes. The two-stage laser treatment design for our study (Figure 1) included the first stage, which was performed once a week for eight weeks, and the second stage, which occurred once every four weeks for four visits. No local anesthesia or cooling device was utilized.

2.3. Treatment Evaluation. Direct microscopy, culture and clinical assessment were performed at $4,8,12,16,20,24$, and 36 weeks. The mycological efficacy was evaluated by the rate of simultaneous negative direct microscopy and negative cultures. Clinical efficacy was divided into four categories: cure (complete new nail growth with a smooth and brightly colored nail plate and less than 5\% defects), significant improvement ( $\geq 60 \%$ new nail growth), improvement $(\geq 20$ and $<60 \%$ new nail growth), and inefficiency $(<20 \%$ new nail growth). The clinical efficacy rate is equal to the cure rate plus the significant improvement rate [7].

Patients were asked to report all adverse events directly during each laser treatment. A five-point Verbal Rating Scale (VRS; $0=$ no pain, $1=$ mild pain, $2=$ moderate pain, $3=$ severe pain, and $4=$ intolerable pain) was used to evaluate pain. Patients were asked to mark the word (one of five that were given) that best fit the pain intensity during the laser treatment.

2.4. Statistic Analysis. An $\chi^{2}$ test was performed to determine the laser efficacy in all nails and to compare the SCIO grades and the degree of hyperkeratosis. P-values of less than 0.05 were considered statistically significance.

\section{Results}

3.1. Patient Characteristics. A total of 100 affected nails (88 toenails and 12 fingernails) from 22 patients ( 8 males and 14 females) were included, of which $37 \%$ were the nail of the big toe. The average age was 49.6 years (range from 27 to 76 years), and only three patients were older than 60 years. These patients exhibited all four clinical types of onychomycosis: distal and lateral subungual onychomycosis (DLSO), superficial white onychomycosis (SWO), proximal subungual onychomycosis (PSO), and total dystrophic onychomycosis (TDO) (Table 1). Overall, $84 \%$ of nails were infected by $T$. rubrum, $8 \%$ were coinfected with T. rubrum and Candida albicans, $4 \%$ were coinfected with T. rubrum and Rhodotorula mucilaginosa, and $4 \%$ were coinfected with T. rubrum and $C$. parapsilosis.

3.2. Mycological Efficacy. Two-stage laser treatment significantly improved the mycological cure rate (negative direct microscopy and negative culture, Figure 2(a)). Week 24 had a significantly higher number of mycological cures than week 8, suggesting the second stage is essential. Regarding each SCIO grade (Figure 3(a)), both SCIO III and SCIO IV were significantly improved at week 24 compared to week 8 , and SCIO II was significantly improved at week 20 compared to week 8 . The efficacy of the laser treatment was correlated with SCIO grade, in that the higher the SCIO grade, the lower the laser efficacy. Compared to the efficacy of SCIO IV, SCIO II had a significantly better efficacy at both week 4 and week 20 . 


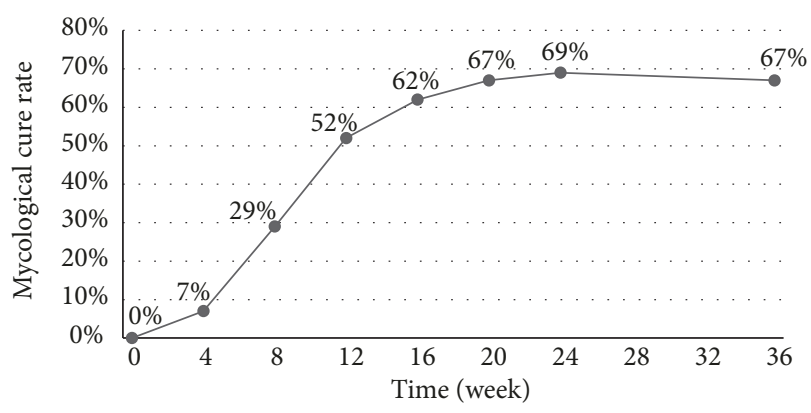

(a)

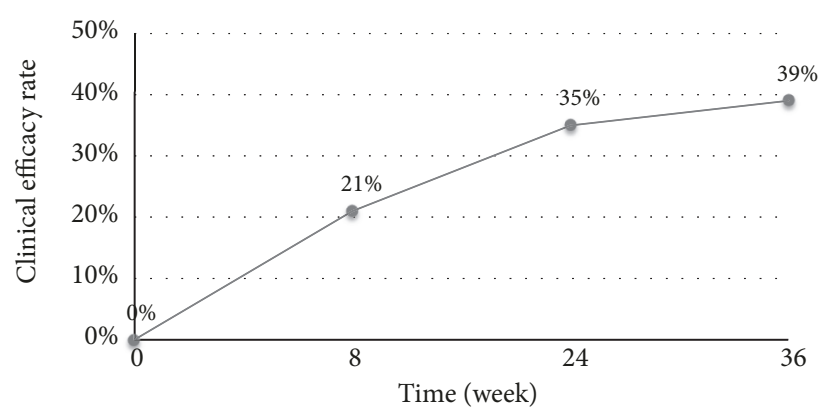

(b)

Figure 2: (a) Mycological cure rate and (b) clinical efficacy rate of nails that received laser treatment. There was a significant improvement in both rates at week 24 , compared to week 8 .

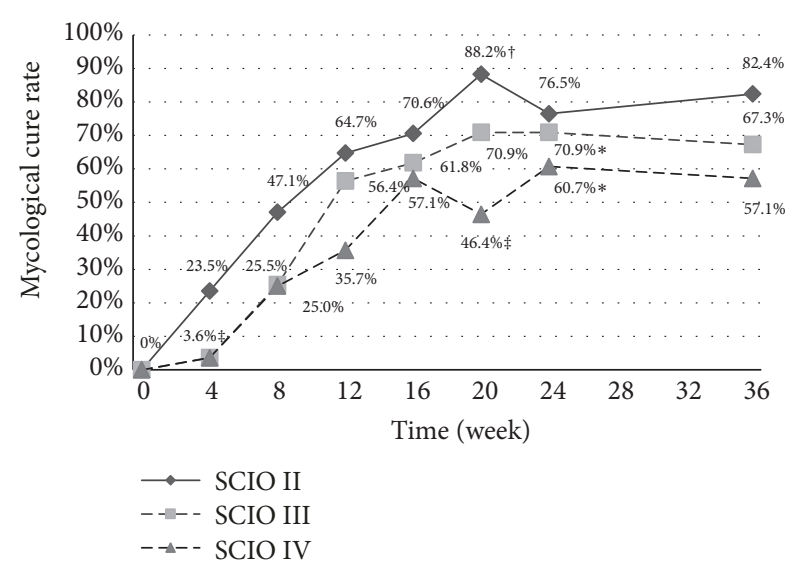

(a)

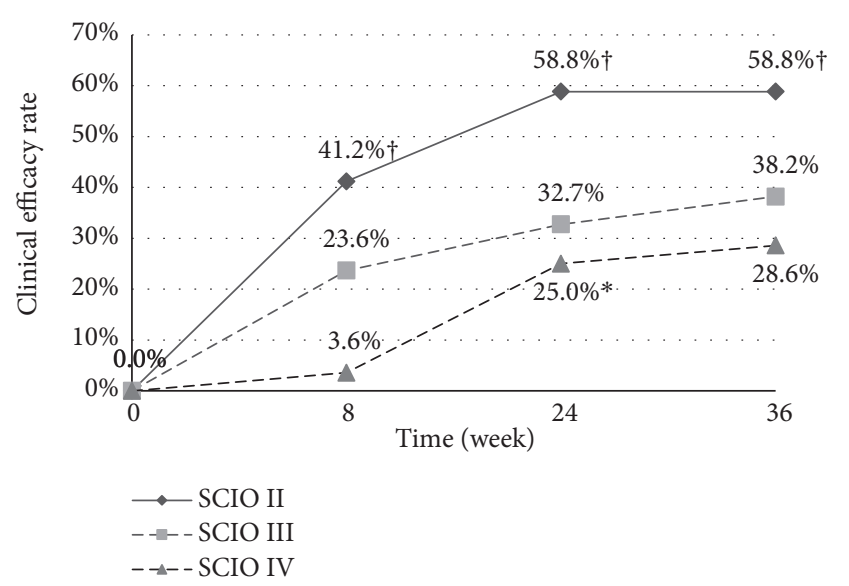

(b)

FIGURE 3: (a) Mycological cure rate of SCIO II, SCIO III, and SCIO IV during laser treatment. $* p<0.05$, comparing week 24 to week 8 . ${ }^{\dagger} p<$ 0.05 , comparing week 20 to week 8 . ${ }^{\ddagger} p<0.05$, comparing SCIO IV to SCIO II at both week 4 and week 20. (b) Clinical efficacy rate of SCIO II, SCIO III, and SCIO IV during laser treatment. $* p<0.05$, comparing week 24 to week $8 .{ }^{\dagger} p<0.05$, comparing SCIO IV to SCIO II at week 8 , week 24 , and week 36 .

TABLE 2: Pain measurement and number of mycologically cured cases at week 36.

\begin{tabular}{lcc}
\hline $\begin{array}{l}\text { Pain level } \\
\text { (Verbal Rating Scale) }\end{array}$ & Number of patients & Number of mycological cures \\
\hline no pain (0) & 0 & 0 \\
mild pain (1) & 3 & $3(100 \%)$ \\
moderate pain (2) & 12 & $8(66.7 \%)$ \\
severe pain (3) & 7 & $4(57.1 \%)$ \\
intolerable pain (4) & 0 & 0 \\
\hline
\end{tabular}

3.3. Clinical Efficacy. The clinical efficacy significantly improved after two-stage laser treatment (Figure 2(b)). Week 24 had significantly higher clinical efficacy rates than week 8. Further, SCIO IV cases significantly improved at week 24 compared to week 8 , and the clinical efficacy of SCIO II was significantly better than for SCIO IV at week 8 , week 24 , and week 36 (Figure 3(b)).

3.4. Pain and Side Effects. All patients reported various intensities of pain using the VRS. Most patients reported moderate pain, while none reported intolerable pain. All patients with mild pain (3 out of 3) were mycologically cured at week 36 , whereas 8 out of 12 with moderate pain and 4 out of 7 with severe pain experienced mycological cures (Table 2). Treatment was tolerated by all subjects, and no side effects were reported at any time during the procedure.

3.5. Treatment Efficacy and Degree of Hyperkeratosis. The mycological cure rate was characterized by the degree of hyperkeratosis. Nails with fungal eradication and hyperkeratosis of less than $1 \mathrm{~mm}$ displayed the best outcomes after laser treatment, with effects starting to appear at week 4 , whereas 


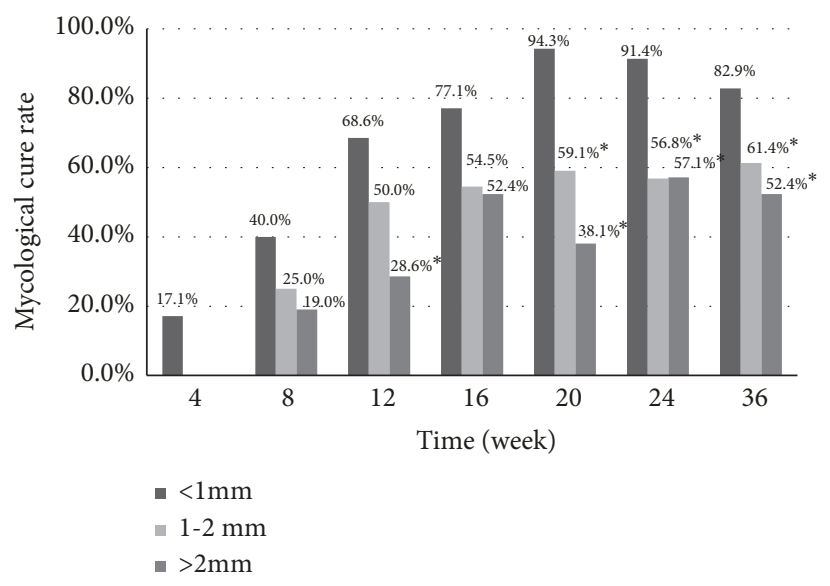

FIGURE 4: Mycological cure rate of hyperkeratosis from weeks 4 to week 36 . The overall outcomes of hyperkeratosis of less than $1 \mathrm{~mm}$ exhibited significant $(* p<0.05)$, which exhibited better effects than those with hyperkeratosis of $1-2 \mathrm{~mm}$ and more than $2 \mathrm{~mm}$ at week 20 , week 24 , and week 36 .

those with hyperkeratosis of 1-2 $\mathrm{mm}$ or greater than $2 \mathrm{~mm}$ saw effects around week 8 . The overall outcomes for nails with hyperkeratosis of less than $1 \mathrm{~mm}$ exhibited significantly better effects than those with hyperkeratosis of 1-2 mm or greater than $2 \mathrm{~mm}$ at week 20 , week 24 , and week 36 (Figure 4).

3.6. Cosmetic Improvement after Laser Treatment and Nail Regrowth. Nail appearances was also evaluated during the procedure. Laser sessions significantly improved cosmetic appearance at week 24 and week 36 (Figure 5), with similar effects being observed in most of the patients.

\section{Discussion}

The clinical treatment of onychomycosis remains extremely challenging due to the high recurrence rate. In general, current clinical interventions include oral, topical, and surgical therapies $[2,5]$. Topical antifungal agents are not efficient in most cases because of the limited penetration of the nail plates. Systemic antifungal agents are considered the standard treatment, but long-term therapy, drug interactions, and side effects often restrain its applications [5-7].

Recently, the Nd:YAG 1064-nm laser was reported to inhibit the in vitro growth of T. rubrum [5, 9, 10]. Laser treatment disrupts the mitochondrial membrane potential by producing reactive oxygen species and inducing apoptosis in fungi [11]. Kozarev et al. [5] treated 194 nails from 72 onychomycosis patients with a long-pulsed $(35 \mathrm{~ms}) \mathrm{Nd}$ :YAG 1064-nm laser (Fotona, 35-40 J/ $\mathrm{cm}^{2}$, spot size $4 \mathrm{~mm}$ ) every week for 4 weeks, and the effects were encouraging. During the 3-month follow-up, $95.83 \%$ of patients had negative cultures. It is worth mentioning that the thicker nails were treated with $40 \%$ urea in this study. Hochman [12] reported negative cultures in $87.5 \%$ of 8 patients treated with a 0.65 ms pulsed Nd:YAG 1064-nm laser (LightPod Neo) using a fluency of $233 \mathrm{~J} / \mathrm{cm}^{2}$ and $2-\mathrm{mm}$ spot size for 2-3 treatments that occurred at least every 3 weeks. An antifungal cream was applied after each treatment.
We designed the long-pulsed Nd:YAG 1064-nm laser (Fotona) with the following parameters: $35-40 \mathrm{~J} / \mathrm{cm}^{2}$, a pulse duration of $35 \mathrm{~ms}$, a spot size of $4 \mathrm{~mm}$, and a frequency $1.0 \mathrm{~Hz}$. The mycological cure rate and the clinical efficacy rate of the nails were $29 \%$ and $21 \%$ at week 8 (first stage of treatment), $69 \%$ and $35 \%$ at week 24 (second stage of treatment), and $67 \%$ and $39 \%$ at week 36 , respectively. The peak mycological efficacy rate occurred at week 24 and was stable until week 36 , suggesting the laser therapy was effective. The peak clinical efficacy rate also occurred at week 24 and continued to increase through week 36 . Long-term follow-up will be needed to determine the clinical recurrence time after laser treatment. Compared to other studies, the severity of onychomycosis, treatment course, treatment interval, parameters of the laser, evaluation criteria, and follow-up time varied, which may contribute to the efficacy of laser treatment. Therefore, randomized, intense follow-up clinical studies, including standard treatments and standard evaluation methods, will be conducted in the future to fully elucidate the efficacy of laser therapy on onychomycosis.

Our study demonstrated that efficacy significantly improved after the second stage of treatment compared to the first stage, suggesting that the second phase was necessary. Compared to other studies with only 8 sessions of laser treatment (one-stage), our study with 12 sessions laser treatment (two-stage) had better mycological efficacy rates. Lu [13] reported both clinical and mycological efficacy rates of $41.3 \%$ at week 36. Zhang [7] reported a clinical efficacy rate of 51\%, with $13 \%$ positive fungal microscopy (100\% at baseline) and $27 \%$ positive fungal culture ( $47 \%$ at baseline) at week 24 . In Min Seok Kim's [14] study, the positive fungal microscopy rate at week 36 was $41.7 \%$ and $42.9 \%$ for groups A and B, respectively, and the positive fungal culture rate was $42.9 \%$ and $40 \%$.

The efficacy of laser treatment also correlated with SCIO grade. Efficacy trend lines revealed that SCIO II was better than SCIO III, that SCIO III was better than SCIO IV, and that there was a significant difference between SCIO II and SCIO IV. Sergeev et al. [8] first published the SCIO to evaluate 


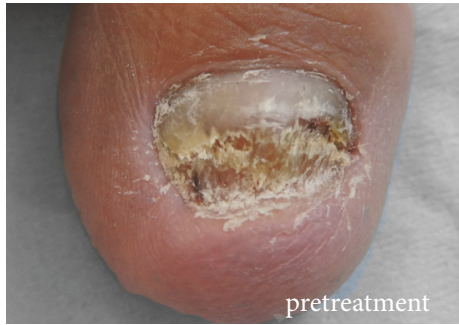

(a)

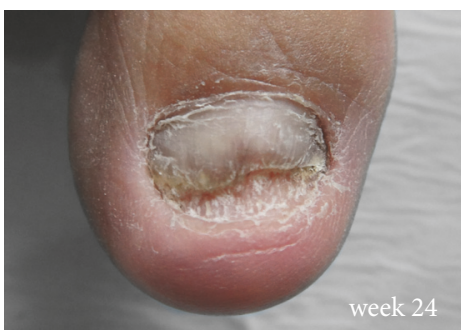

(c)

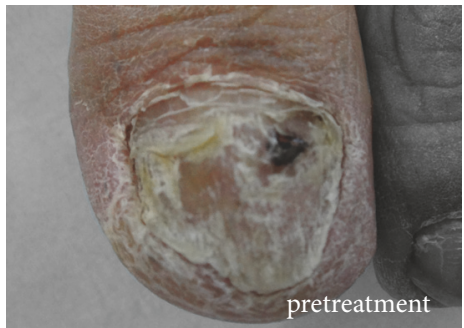

(e)

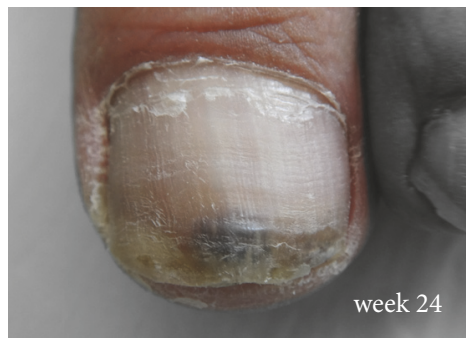

(g)

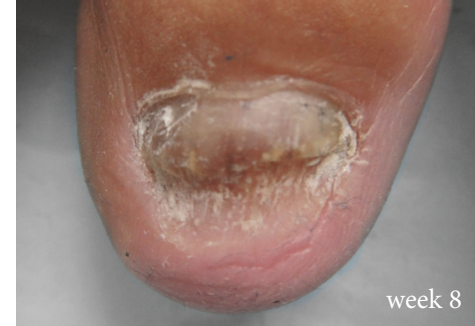

(b)

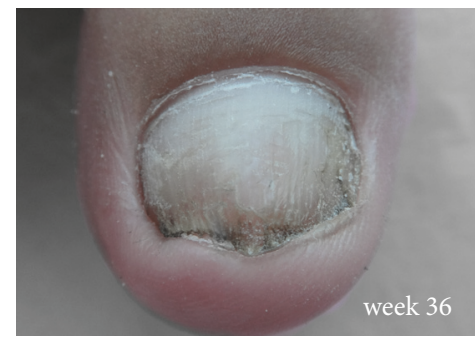

(d)

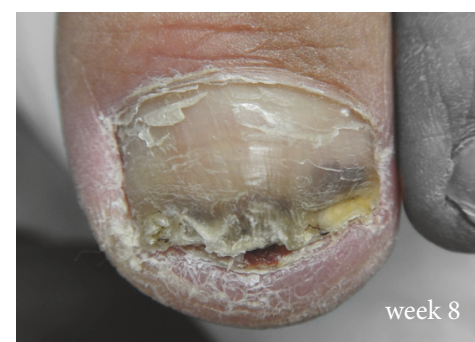

(f)

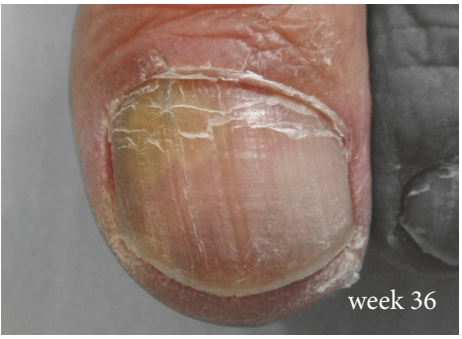

(h)

FIGURE 5: Representative photographs of the nails before and after laser treatment from two patients. Patient 1: (a) pretreatment, (b) week 8 , (c) week 24, and (d) week 36. Patient 2: (e) pretreatment, (f) week 8, (g) week 24, and (h) week 36.

the severity of onychomycosis. Our results indicate that the SCIO may be used to provide laser treatment strategies: SCIO II and SCIO III patients should receive laser therapy alone (our team found the efficacy of systemic antifungals was equal to laser therapy in those patients [15]); SCIO IV patients should receive combination therapy (systemic antifungals, laser therapy, and topical therapy); and SCIO IV patients need a longer laser treatment course than SCIO II and SCIO III patients.

Regarding the degree of hyperkeratosis, we found that nails with hyperkeratosis of less than $1 \mathrm{~mm}$ had better outcomes. If nails thicker than $1 \mathrm{~mm}$ are treated with $40 \%$ urea before laser treatment, it could improve the overall efficacy.
In total, 84 nails from 19 patients were infected by $T$. rubrum, and 16 nails of 3 patients were coinfected with $T$. rubrum and other fugal strains. The mycological cure rate and clinical efficacy rate of nails with T. rubrum infection were $35 \%$ and $25 \%$ at week 8 (first stage of treatment), $69 \%$ and $38 \%$ at week 24 (second stage of treatment), and $68 \%$ and $45 \%$ at week 36, respectively. The mycological cure rate and clinical efficacy rate of nails with mixed fungal infections were $0 \%$ and $0 \%$ at week $8,69 \%$ and $6.25 \%$ at week 24 , and $63 \%$ and $6.25 \%$ at week 36 , respectively. The mycological and clinical efficacy of laser treatment for nails with mixed fungal infections was much lower than the nails with T. rubrum infection alone. T. rubrum seemed to be more sensitive to laser intervention, 
possibly due to it containing more pigment than other fungi, such as C. albicans [16].

All patients experienced mild to severe pain during laser treatment, and thus, patients with high pain tolerance had better laser treatment efficacy. Laser therapy uses heat energy, which is the reason why the pain tolerant patients saw better outcomes. The question of whether it is necessary to use cooling device or anesthesia before laser treatment to get better effects is difficult to answer. Cooling devices or anesthesia may help patients to withstand the pain associated with treatment, so as to improve the laser effect, but there have been reports of nail loss as a result of overheating [11]. Further studies on the safety of laser therapy on the nail matrix need to be examined in depth.

\section{Conclusions}

In conclusion, two-stage long-pulsed Nd:YAG 1064-nm laser treatment exhibited an encouraging efficacy and safety modality in patients with onychomycosis.

\section{Data Availability}

The datasets generated and analyzed during this study are available from the corresponding author upon reasonable request.

\section{Conflicts of Interest}

There are no financial or other conflicts of interest.

\section{References}

[1] B. E. Elewski and M. A. Charif, "Prevalence of onychomycosis in patients attending a dermatology clinic in northeastern Ohio for other conditions," Archives of dermatology, vol. 133, no. 9, pp. 1172-1173, 1997.

[2] J. J. Finch and E. M. Warshaw, "Toenail onychomycosis: current and future treatment options," Dermatologic Therapy, vol. 20, no. 1, pp. 31-46, 2007.

[3] D. P. Lubeck, "Measuring health-related quality of life in onychomycosis," Journal of the American Academy of Dermatology, vol. 38, no. 5, pp. S64-S68, 1998.

[4] A. Shemer, "Update: Medical treatment of onychomycosis," Dermatologic Therapy, vol. 25, no. 6, pp. 582-593, 2012.

[5] J. V. Z. Kozarev, "Novel laser therapy in treatment of onychomycosis," Journal - Laser \& Health Academy, vol. 1, pp. 1-8, 2010.

[6] U. Kimura, K. Takeuchi, A. Kinoshita, K. Takamori, M. Hiruma, and Y. Suga, "Treating onychomycoses of the toenail: Clinical efficacy of the sub-millisecond 1,064 nm Nd: YAG laser using a $5 \mathrm{~mm}$ spot diameter," Journal of Drugs in Dermatology (JDD), vol. 11, no. 4, pp. 496-504, 2012.

[7] R. N. Zhang, D. K. Wang, F. L. Zhuo, X. H. Duan, X. Y. Zhang, and J. Y. Zhao, "Long-pulse Nd:YAG 1064-nm laser treatment for onychomycosis," Chinese medical journal, vol. 125, no. 18, pp. 3288-3291, 2012.

[8] A. Y. Sergeev, A. K. Gupta, and Y. V. Sergeev, "The scoring clinical index for onychomycosis (SCIO index)," Skin Therapy Letter, vol. 7, pp. 6-7, 2002.

[9] E. Vural, H. L. Winfield, A. W. Shingleton, T. D. Horn, and G. Shafirstein, "The effects of laser irradiation on Trichophyton rubrum growth," Lasers in Medical Science, vol. 23, no. 4, pp. 349-353, 2008.

[10] Z. L. Xu, J. Xu, FL. Zhuo et al., "Effects of laser irradiation on Trichophyton rubrum growth and ultrastructure," Chinese medical journal, vol. 125, no. 20, pp. 3697-3700, 2012.

[11] P. Nenoff, S. Grunewald, and U. Paasch, "Laser therapy of onychomycosis," Journal of the German Society of Dermatology : JDDG, vol. 12, no. 1, pp. 33-38, 2014.

[12] L. G. Hochman, "Laser treatment of onychomycosis using a novel 0.65 -millisecond pulsed Nd:YAG 1064-nm laser," Journal of Cosmetic and Laser Therapy, vol. 13, no. 1, pp. 2-5, 2011.

[13] S. Lu, J. Zhang, Y. Liang, X. Li, W. Cai, and L. Xi, “The efficacy and prognostic factors for long pulse neodymium: yttriumaluminum-garnet laser treatment on onychomycosis: a pilot study," Annals of Dermatology, vol. 28, no. 3, pp. 406-408, 2016.

[14] M. S. Kim, J. Y. Jung, E. B. Cho, E. J. Park, K. H. Kim, and K. J. Kim, "The effectiveness of 1,064-nm long-pulsed Nd:YAG laser in the treatment of severe onychomycosis," Journal of Cosmetic and Laser Therapy, vol. 18, no. 6, pp. 317-322, 2016.

[15] Y. Li, J. Xu, J.-Y. Zhao, and F.-L. Zhuo, "Self-controlled study of onychomycosis treated with long-pulsed Nd:YAG 1064-nm laser combined with itraconazole," Chinese Medical Journal, vol. 129, no. 16, pp. 1929-1934, 2016.

[16] Y. Li, S. Yu, J. Xu, R. Zhang, and J. Zhao, "Comparison of the efficacy of long-pulsed Nd:YAG laser intervention for treatment of onychomycosis of toenails or fingernails," Journal of Drugs in Dermatology: JDD, vol. 13, no. 10, pp. 1258-1263, 2014. 


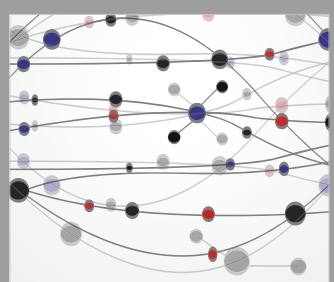

The Scientific World Journal
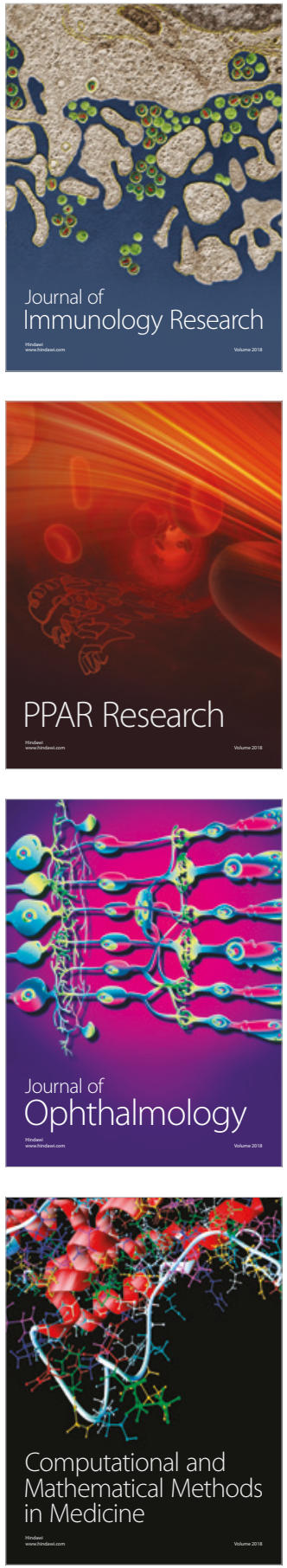

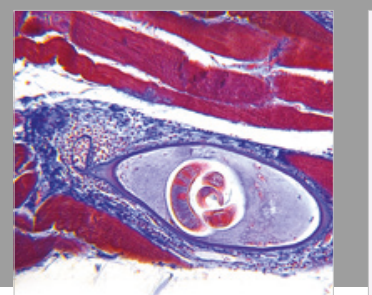

Gastroenterology Research and Practice

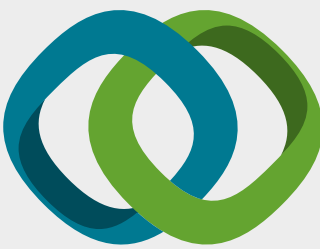

\section{Hindawi}

Submit your manuscripts at

www.hindawi.com
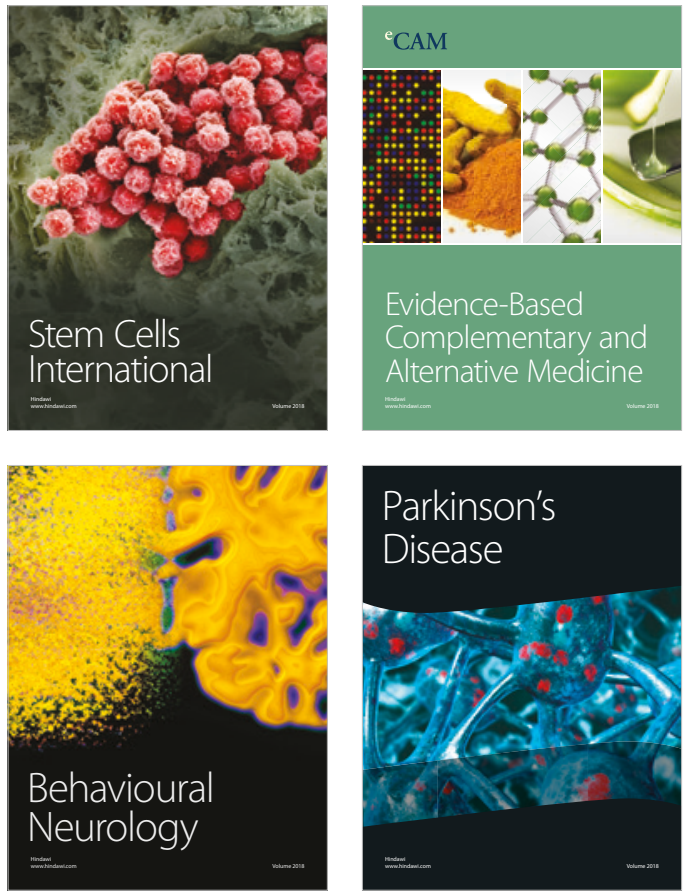

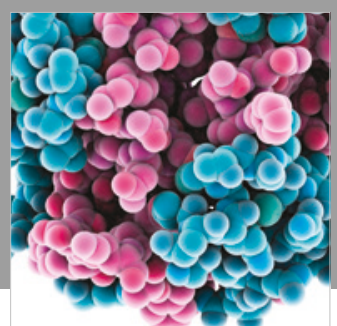

ournal of

Diabetes Research

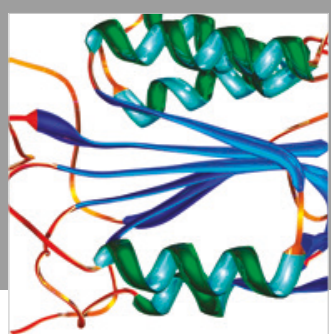

Disease Markers
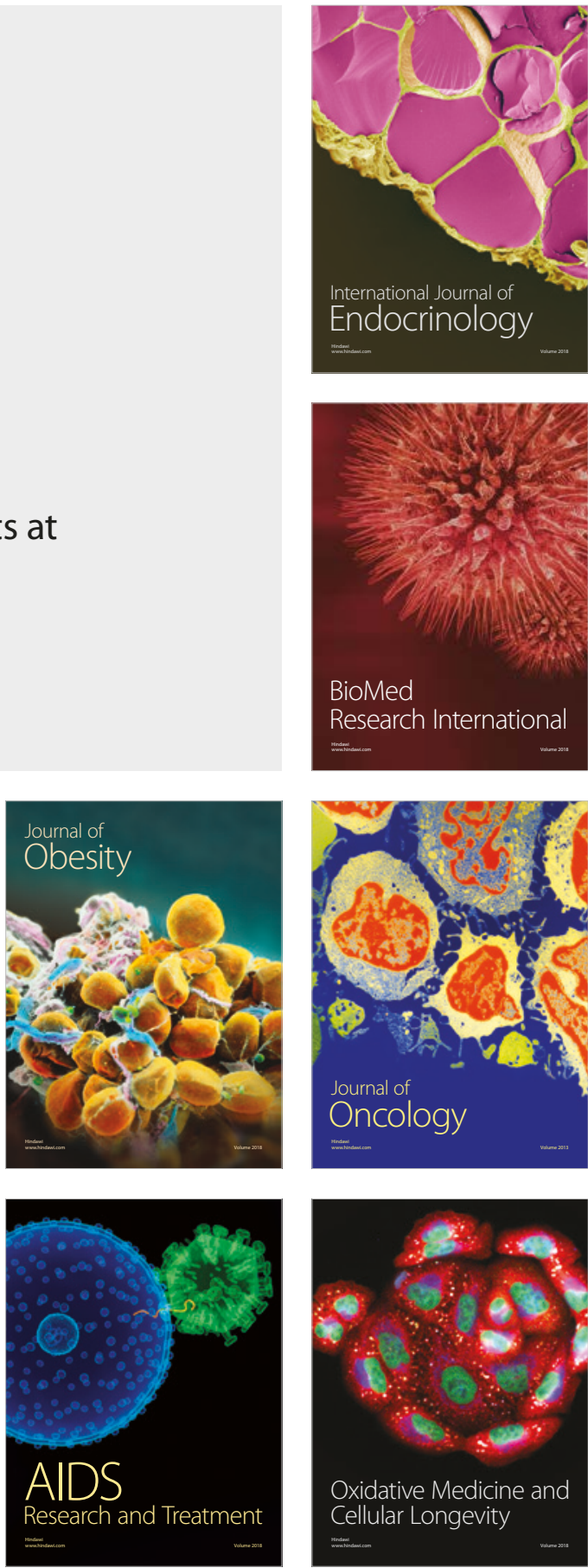